\title{
LYAPUNOV STABILITY VIA DIFFERENTIAL MOMENTS
}

\author{
BY \\ R. J. CHARRON \\ Memorial University of Newfoundland, St. John's, Newfoundland, Canada
}

\begin{abstract}
We show how differential moments can be used to construct Lyapunov functions for general, autonomous and nonautonomous, second- and third-degree ordinary differential equations. In certain instances, one can extend classical results to sequences of Lyapunov functions.
\end{abstract}

1. Introduction. In applying Lyapunov's direct (second) method to problems of stability of solutions to an ordinary differential equation $L[\mathbf{x}]=0$, where $\mathbf{x}:=$ $\left(x(t), \dot{x}(t), \ldots, x^{n}(t)\right)$, one normally constructs a single function, say $v(\mathbf{x}, t)$, which is sign definite, differentiable (with respect to $t$ ), and such that $v_{t}(\mathbf{x}, t)$ is also sign definite (semi-definite) with sign opposite to that of $v(\mathbf{x}, t)$. There is, however, no systematic procedure for the construction of Lyapunov functions. Our constructions here should partially redress this situation.

We will in the remainder of this article interchangeably denote the differential equation of interest in operator form as $L[\mathbf{x}]=0$ or as $\dot{\mathbf{x}}=f(\mathbf{x}, t)$. In either case, the implication is that the origin is an equilibrium point and we assume $f(\mathbf{x}, t)$ is sufficiently smooth in $\Omega:=[0, \infty) \times \mathbb{R}^{n}$ so that for any $\left(\mathbf{x}_{0}, t_{0}\right) \in \Omega$ there passes a unique solution, denoted by $x\left(t ; \mathbf{x}_{0}, t_{0}\right)$, of the differential equation. As it is customary, we only analyze the stability of the null solution to $L[\mathbf{x}]=0$.

Definition. The null solution of $L[\mathbf{x}]=0$ is stable if for any $\varepsilon>0$ and $t_{0} \in[0, \infty), \exists \delta\left(\varepsilon, t_{0}\right)>0$ such that for all $\mathbf{x}_{0}$ with $\left\|\mathbf{x}_{0}\right\|<\delta$ and $t \in\left[t_{0}, \infty\right)$, $\left\|x\left(t ; \mathbf{x}_{0}, t_{0}\right)\right\|<\varepsilon$. If $\delta$ is independent of $t_{0}$, then the null solution is uniformly stable.

Definition. The null solution of $L[\mathbf{x}]=0$ is asymptotically stable if it is stable and $x\left(t ; \mathbf{x}_{0}, t_{0}\right) \rightarrow 0$ as $t \rightarrow \infty$.

TheOREM 1.1 (Lyapunov, 1982). The null solution of $L[\mathbf{x}]=0$ is stable if there exists for $(t, \mathbf{x}) \in \Omega$ a positive definite function $v(\mathbf{x}, t)$ such that $v(\mathbf{0}, t)=0$ and $v_{t}(\mathbf{x}, t)$ is negative semi-definite.

TheOREM 1.2 (Persidski, 1933). The null solution of $L[\mathbf{x}]=0$ is uniformly stable if there exists for $(t, \mathbf{x}) \in \Omega$ a uniformly bounded, positive definite function $v(\mathbf{x}, t)$

Received December 1, 1989.

1991 Mathematics Subject Classification. Primary 34D.

This work was supported in part by the Natural Sciences and Engineering Research Council of Canada.

(C)1991 Brown University 
such that $v(\mathbf{0}, t)=0$ and $v_{t}(\mathbf{x}, t)$ is negative semidefinite.

Definition (Lyapunov). $v(\mathbf{x}, t)$ has an infinitely small upper bound if for any $\varepsilon>0, \exists \delta>0$ such that if $\|\mathbf{x}\|<\delta$ and $t \geq 0$, then $|v(\mathbf{x}, t)|<\varepsilon$.

THEOREM 1.3 (Lyapunov, 1892). The null solution of $L[\mathbf{x}]=0$ is asymptotically stable if there exists for $(t, \mathbf{x}) \in \Omega$ a positive definite function $v(\mathbf{x}, t)$ which has infinitely small upper bound and is such that $v_{t}(\mathbf{x}, t)$ is negative definite.

RemarK. The asymptotic stability is in fact global when $v(\mathbf{x}, t) \rightarrow \infty$ as $\|\mathbf{x}\|_{2} \rightarrow$ $\infty$.

Ponzo [11, 12] introduced the notion of differential moments, hereafter denoted $M_{n}$, defined as

$$
M_{n}:=\int_{t_{0}}^{t} x^{(n)} L[x] d s
$$

and employed them to resolve questions of stability for certain linear and nonlinear differential equations. In the following sections, we will extend Ponzo's initiatives to general second- and third-order equations.

2. Main results. We consider, in this section, general, second-order differential equations

$$
L_{2}[x]:=\ddot{x}+a(x, \dot{x}, t) \dot{x}+b(x, t) x=0
$$

where $a(x, \dot{x}, t)$ and $b(x, t)$ are at least $C(\Omega)$. Our definition of $L_{2}[x]$ and that of the differential moments $M_{n}$ implies $M_{n}=0 \forall n$. As a general rule, from each equation $M_{n}=0$ we will integrate (by parts or otherwise) the terms $\int_{t_{0}}^{t} x^{(n)} \ddot{x} d t$ and leave the remaining terms unchanged. By taking linear combinations of the resulting relations we will construct our stability results. This is done in a systematic way to generate quadratic forms for both $v(\mathbf{x}, t)$ and $v_{t}(\mathbf{x}, t)$. The stability results will then follow by applying Sylvester's criterion for sign definiteness of quadratic forms.

THEOREM 2.1. $L_{2}[x]$ has a uniformly stable null solution if there exists constant $\alpha, \beta$ with $4 \beta>\alpha^{2}$ such that

(i) $a(x, \dot{x}, t)>\alpha / 2>0$,

(ii) $b(x, t)>0$,

(iii) $(2 b(x, t)+\alpha a(x, \dot{x}, t)-2 \beta)^{2}<4 \alpha b(x, t)(2 a(x, \dot{x}, t)-\alpha)$.

If, furthermore, $L_{2}[x]$ is autonomous then the null solution of $L_{2}[x]$ is globally asymptotically stable.

Proof. For ease of exposition, we will drop explicit mention of the dependence of $a(x, \dot{x}, t)$ and $b(x, t)$ on their dependent variables. Furthermore, $M_{0}=0$ implies the relation

$$
\left.x \dot{x}\right|_{t_{0}} ^{t}=\int_{t_{0}}^{t}\left(\dot{x}^{2}-a x \dot{x}-b x^{2}\right) d s
$$

and likewise $M_{1}=0$ implies

$$
\left.\dot{x}^{2}\right|_{t_{0}} ^{t}=-2 \int_{t_{0}}^{t}\left(a \dot{x}^{2}+b x \dot{x}\right) d s .
$$


Now let $\alpha$ and $\beta$ be two nonzero real numbers such that $\alpha^{2}<4 \beta$ and form

$$
v(x, \dot{x} ; \alpha, \beta)=\dot{x}^{2}+\alpha x \dot{x}+\beta x^{2}
$$

which is now positive definite. From (2.1), (2.2), and noting that $\left.x^{2}\right|_{t_{0}} ^{t}=2 \int_{t_{0}}^{t} x \dot{x} d s$, we also have

$$
\frac{d}{d t} v(x, \dot{x} ; \alpha, \beta)=(\alpha-2 a) \dot{x}^{2}+(2 \beta-2 b-\alpha a) x \dot{x}-\alpha b x^{2},
$$

so that if conditions (i)-(iii) of the hypothesis hold, then $v_{t}(x, \dot{x} ; \alpha, \beta)$ is negative semi-definite. The null solution is the only solution to $L[x]=0$ such that $v(x, \dot{x} ; \alpha, \beta)$ and $v_{t}(x, \dot{x} ; \alpha, \beta)$ are zero, and furthermore $v(x, \dot{x} ; \alpha, \beta)$ is both uniformly bounded and has infinitely small upper bound. Finally, when $L_{2}[x]$ is autonomous $v_{t}(x, \dot{x} ; \alpha, \beta)$ is negative definite. Theorems (1.1)-(1.3) conclude the proof.

REMARK. When applying this technique to specific problems it may of course be advantageous to work with different arrangements from each $M_{n}$. For instance, in studying the stability of

$$
\ddot{x}+q \dot{x}+p(t) x=0
$$

([3]), one can obtain from $M_{0}=0$ and $M_{1}=0$ the relations

$$
2 x \dot{x}+\left.q x^{2}\right|_{t_{0}} ^{t}=\int_{t_{0}}^{t}\left(2 \dot{x}^{2}-2 p(t) x^{2}\right) d s
$$

and

$$
\dot{x}^{2}+\left.p x^{2}\right|_{t_{0}} ^{t}=-\int_{t_{0}}^{t}\left(2 q \dot{x}^{2}-\dot{p} x^{2}\right) d s .
$$

From the combination $M_{1}+\alpha M_{0}$, we can go on to conclude the null solution is uniformly globally asymptotically stable provided $p(t)>0, q>\alpha$ and there exists a constant $\beta$ such that

$$
2 p(t)-\frac{\dot{p}}{\alpha} \geq \beta>0 .
$$

Within the constraint imposed on $\alpha$ and $\beta$ there is much insight to be gained by understanding the geometric nature, in the $a b$-plane, of condition (iii) in the hypothesis. The coordinate transformation

$$
\left(\begin{array}{l}
a \\
b
\end{array}\right)=\frac{1}{\sqrt{4+\alpha^{2}}}\left(\begin{array}{cc}
2 & -\alpha \\
\alpha & 2
\end{array}\right)\left(\begin{array}{l}
u \\
v
\end{array}\right)
$$

allows us to rewrite (iii) in its canonical form in the corresponding $u v$-plane as

$$
v^{2}+\phi v+\psi u+\gamma<0
$$

where

$$
\phi=\frac{4 \alpha^{2}(2+\beta)-16 \beta}{\left(4+\alpha^{2}\right)^{3 / 2}}, \quad \psi=\frac{4 \alpha\left(\alpha^{2}-4 \beta\right)}{\left(4+\alpha^{2}\right)^{3 / 2}}, \quad \gamma=\frac{4 \beta^{2}}{4+\alpha^{2}} .
$$

Thus the region defined by (i)-(iii) with $\alpha^{2}<4 \beta$, hereafter denoted $\Theta_{\alpha, \beta}$, is the interior of a parabola wholly contained in the intersection of the planes $a>0, b>0$ 
when $\alpha>0$, and is an empty set when $\alpha<0$. Furthermore each $\Theta_{\alpha, \beta}$ is strictly convex and $\Xi:=\left\{\bigcup \Theta_{\alpha, \beta}: \alpha \in \mathbb{R}^{+}, \alpha^{2} / 4<\beta\right\}$ forms, in the $a b$-plane, an open covering of the region bounded by $a>\alpha$ and $b>0$. This follows by considering the envelope of the curves generated by the left-hand side of (2.3) with $\beta$ variable and holding $\alpha$ to a fixed, but arbitrarily small quantity. Thus the classical result of Routh and Hurwitz for the case where $a(x, \dot{x}, t)$ and $b(x, t)$ are constant follows as a special case. This same result now also holds when only $b(x, t)$ is constant.

Example. Consider the damped Mathieu equation

$$
\ddot{x}+2 \zeta \dot{x}+(c-2 q \cos (2 t)) x=0
$$

where $\zeta$ and $c$ are positive. With $b(x, t):=c-2 q \cos (2 t)$, it is of interest to specify an upper bound on $q$ so as to guarantee stability of the null solution. From theorem (2.1), we simply need to find the optimum $\alpha, \beta$ such that the largest possible segment of the line $l$ from $(2 \zeta, 0)$ to $(2 \zeta, 2 c)$ in the $a b$-plane will be contained in the parabola $\Theta_{\alpha, \beta}$. Let $b_{1}$ and $b_{2}$ (with $b_{1}>b_{2}$ ) be the intercepts of $l$ with $\Theta_{\alpha, \beta}$. The oscillatory nature of $b(x, t)$ suggests the optimum solution will satisfy $2 c-b_{1}=b_{2}$ which implies $\beta=\alpha^{2} / 2+c-\alpha \zeta$. From this point, it is not too difficult to find the optimal solution for $c>2 \zeta^{2}$ to require $\alpha=2 \zeta$, whence uniform stability of the null solution for $q<\zeta \sqrt{c-\zeta^{2}}$. This reproduces the result of Micheal [6]. We remark that Parks [9], Narendra and Taylor [7], and Ramarajan and Rao [13] have all considered the stability of the null solution to (2.4), and have obtained less restrictive results particularly for $c \gg \zeta^{2}$.

The geometry of (i)-(iii) as detailed above does raise the interesting question of whether condition (iii) is at all necessary. The answer to this question is affirmative. To see this, we associate with each $v(\mathbf{x} ; \alpha, \beta)$ two sets: the region $\Theta_{\alpha, \beta}$ of the $a b$-plane where $v_{t}(\mathbf{x} ; \alpha, \beta)$ is negative semi-definite and the ellipse in the $x \dot{x}$-plane defined by $\Omega_{\mathbf{x}_{0}, \alpha, \beta}:=\left\{\mathbf{x} \mid v(\mathbf{x} ; \alpha, \beta) \leq v\left(\mathbf{x}_{0} ; \alpha, \beta\right)\right\}$. Assume $(a(x, \dot{x}, t), b(x, t))$ for $t \geq 0$ traces a curve in the first quadrant of the $a b$ plane such that $(a(x, \dot{x}, t), b(x, t)) \in \bigcup_{n}\left\{\Theta_{\alpha, \beta_{n}}\right\}$ for some $\alpha$ fixed and $\left\{\beta_{n}\right\}_{n=0}^{\infty}$. For each $n$, there is therefore an interval $\left[t_{n}, t_{n+1}\right)$ for which $v(\mathbf{x}, t)$ is positive definite and $v_{t}(\mathbf{x}, t)$ is negative semi-definite. For each $n, v\left(\mathbf{x}\left(t_{n+1}\right) ; \alpha, \beta_{n}\right)<$ $v\left(\mathbf{x}\left(t_{n}\right) ; \alpha, \beta_{n}\right)$ implies $\mathbf{x}(t) \in \Omega_{\mathbf{x}\left(t_{n}\right), \alpha, \beta_{n}}$ for $t \in\left[t_{n}, t_{n+1}\right)$. But the ellipses $\Omega_{\varepsilon, \alpha, \beta_{n}}$ for any fixed $\varepsilon$ can include points $\mathbf{x}$ with $\|\mathbf{x}\|$ arbitrarily large with the appropriate selection of $\beta_{n}$. In general then, given any $\left\|\mathbf{x}_{0}\right\|<\varepsilon$, the solution $x\left(t ; x_{0}, t_{0}\right)$ cannot be bounded away from infinity simply by selecting a sequence of Lyapunov functions each of which are applicable only to a finite portion of the overall trajectory. There is, of course one exception to the rule and we include it here without proof.

Corollary 2.1. Let $\alpha \in \mathbb{R}^{+},\left\{\beta_{m}\right\}_{m=0}^{n}$ be a sequence of real numbers such that $\alpha^{2}<4 \beta_{m}$ for $m=0, \ldots, n$, and $\left\{t_{m}\right\}_{m=0}^{n-1}$ be a strictly increasing sequence of positive real numbers. If $(a(x, \dot{x}, t), b(x, t)) \in \Theta_{\alpha, \beta_{m}}$ for $t \in\left[t_{m}, t_{m+1}\right)$ with 
$m=0, \ldots, n-1$ and $(a(x, \dot{x}, t), b(x, t)) \in \Theta_{\alpha, \beta_{n}}$ for $t \geq t_{n}$ then

(i) The null solution of $L_{2}[x]$ is uniformly stable when $L_{2}[x]$ is nonautonomous.

(ii) The null solution of $L_{2}[x]$ is globally asymptotically stable when $L_{2}[x]$ is autonomous.

We go on now to consider the general third-order problems

$$
L_{3}[x]:=x^{(3)}+a(x, \dot{x}, \ddot{x}, t) \ddot{x}+b(x, \dot{x}, t) \dot{x}+c(x, t) x=0
$$

where $c(x, t) \in C^{1}(\Omega)$. The special case where $c(x, t)$ is a constant will be treated as a separate item later on. For ease of exposition, we label $\dot{x}=y$ and $\dot{y}=z$ so that from $M_{1}=0$ we obtain the relation

$$
y z+\left.c(x, s) \frac{x^{2}}{2}\right|_{t_{0}} ^{t}=\int_{t_{0}}^{t}\left(z^{2}-a y z-b y^{2}+\dot{c} \frac{x^{2}}{2}\right) d s .
$$

We also have from $M_{2}=0$

$$
\left.\frac{z^{2}}{2}\right|_{t_{0}} ^{t}=-\int_{t_{0}}^{t}\left(a z^{2}+b y z+c x y\right) d s .
$$

Thus the linear combination $M_{2}+\alpha M_{1}+\beta y^{2}$ generates the relation

$$
\begin{aligned}
z^{2}+ & \alpha y z+\frac{\alpha}{2} c(x, s) \frac{x^{2}}{2}+\left.\beta y^{2}\right|_{t_{0}} ^{t} \\
& =-\int_{t_{0}}^{t}\left((2 a-\alpha) z^{2}+(2 b-2 \beta+\alpha a) y z+\alpha b y^{2}-\frac{\alpha}{2} \dot{c} x^{2}+2 c x z\right) d s .
\end{aligned}
$$

From the above, we define

$$
v(x, y, z, t ; \alpha, \beta)=z^{2}+\alpha y z+\frac{\alpha}{2} c(x, t) x^{2}+\beta y^{2}
$$

which is positive definite provided $\alpha>0,4 \beta>\alpha^{2}$, and $c(x, t)>0$. Applying Sylvester's criterion to $v_{t}(\mathbf{x} ; \alpha, \beta)$ we can then establish

THEOREM 2.2. The null solution of $L_{3}[x]$ is uniformly stable if there exists constants $\alpha, \beta, \gamma$ with $4 \beta>\alpha^{2}$ such that

(i) $2 a(x, \dot{x}, \ddot{x}, t)>\alpha$,

(ii) $b(x, \dot{x}, t)>0$,

(iii) $c(x, t)>0$,

(iv) $(2 b(x, \dot{x}, t)-2 \beta+\alpha a(x, \dot{x}, \ddot{x}, t))^{2}<4 \alpha b(x, \dot{x}, t)(2 a(x, \dot{x}, \ddot{x}, t)-\alpha)-\gamma$,

(v) $\dot{c}(x, t)<-\frac{8}{\gamma} b(x, \dot{x}, t) c^{2}(x, t)$.

If furthermore, $L_{3}[x]$ is autonomous, then the null solution of $L_{3}[x]$ is globally asymptotically stable.

REMARKS.

(i) As with the corresponding result for $L_{2}[x]$ it is in fact sufficient that conditions (iv) and (v) hold not for all $t$ but rather for all $t>t_{1}$ ( $t_{1}$ arbitrary, but fixed). 
(ii) Compare this result, for instance, to those of Narendra and Goldwyn [8], Simanov [15], Ezeilo [2], and Barbashin [1].

Finally, the special case where $c(x, t)$ is constant yields a result which is much closer to the classical result of Routh and Hurwitz for third-order equations with constant coefficients. (These are: $a>0, b>0, c>0, a b-c>0$.) It follows directly from the linear combination $M_{2}+\alpha M_{1}+\gamma y^{2}$.

Corollary 2.2. The null solution of $L_{3}[x]$ with $c(x, t)$ constant is uniformly stable if there exist constants $\alpha, \delta, \gamma$ with $4 \gamma>\alpha^{2}+8 c / \alpha$ such that

(i) $2 a(x, \dot{x}, \ddot{x}, t)>\alpha$,

(ii) $b(x, \dot{x}, t)>0$,

(iii) $c>0$,

(iv) $4 \delta(2 a(x, \dot{x}, \ddot{x}, t)-\alpha)>(2 b(x, \dot{x}, t)-2 \gamma+\alpha a(x, \dot{x}, \ddot{x}, t))^{2}$,

(v) $\alpha b(x, \dot{x}, t)-2 c>\delta$.

If furthermore, $L_{3}[x]$ is autonomous, then the null solution of $L_{3}[x]$ is globally asymptotically stable.

Acknowlegment. The author acknowledges the benefit of helpful discussions with Dr. Peter Ponzo of the University of Waterloo, as well as helpful suggestions of one of the referees.

\section{REFERENCES}

[1] E. A. Barbashin, On the stability of solution of a third order nonlinear differential equation, Prik. Math. Mekh. 16, 629-632 (1952)

[2] J. O. C. Ezeilo, On the stability of solutions of certain differential equations of the third order, Quart. J. Math. 11, 64-69 (1960)

[3] G. K. Kulev and D. D. Bainov, On the asymptotic stability of systems with impulses by the direct method of Lyapunov, J. Math. Anal. Appl. 140, 324-340 (1989)

[4] H. Leipholz, Stability Theory, Academic Press, New York, 1970

[5] A. M. Lyapunov, Probleme générale de la stabilité du mouvement, Annals of Math. Studies, Vol. 17, Princeton University Press, Princeton, New Jersey, 1949

[6] G. J. Michael, Explicit stability criteria for the damped Mathieu equation, IEEE Trans. Automat. Control AC-12, 337-338 (1967)

[7] K. S. Narendra and J. H. Taylor, Stability of the damped Mathieu equation, IEEE Trans. Automat. Control AC-13, 726 (1968)

[8] K. S. Narendra and R. M. Goldwyn, Stability of certain nonlinear differential equations, IEEE Trans. Automat. Control AC-8, 381-382 (1963)

[9] P. C. Parks, Comments on 'Explicit stability criteria for the damped Mathieu equation', IEEE Trans. Automat. Control AC-13, 129 (1968)

[10] K. P. Persidski, On the stability of motion in first approximation, Mat. Sb. 40, 284-293 (1933)

[11] P. J. Ponzo, On the Stability of certain nonlinear differential equations, IEEE Trans. Automat. Control AC-19, 470-472 (1965)

[12] P. J. Ponzo, Some stability conditions for linear differential equations, IEEE Trans. Automat. Control AC-13, 721-722 (1968)

[13] S. Ramarajan and S. N. Rao, An improved stability criteria for the damped Mathieu equation, IEEE Trans. Automat. Control AC-16, 363-364 (1971)

[14] N. Rouche, P. Habets, and M. Laloy, Stability Theory by Lyapunov's Direct Method, Applied Mathematical Sciences, Vol. 22, Springer-Verlag, New York, 1977

[15] S. N. Simanov, On the stability of solution of a nonlinear equation of the third order, Akad. Nauk. SSSR, Prikl. Mat. Mekh. 17, 369-372 (1953) (in Russian) 